\title{
Free-surface optical scattering as an indicator of the shock- induced solid-liquid phase transition in tin
}

\author{
G. D. Stevens, ${ }^{1}$ S. S. Lutz, B. R. Marshall, W. D. Turley, and L. R. Veeser \\ National Security Technologies, LLC, Special Technologies Laboratory, Santa Barbara, CA 93111
}

\author{
M. R. Furlanetto, R. S. Hixson, D. B. Holtkamp, B. J. Jensen, P. A. Rigg, and M. D. Wilke \\ Los Alamos National Laboratory, Los Alamos, NM 87545
}

\begin{abstract}
When highly polished metal surfaces melt upon release after shock loading, they exhibit features that suggest significant surface changes accompany the phase transition. The reflection of light from such surfaces changes from specular (pre-shock) to diffuse upon melting. A familiar manifestation of this phenomenon is the loss of signal light in VISAR measurements, which occurs at pressures high enough to melt the free surface. Unlike many other potential material phase-sensitive diagnostics (e.g., reflectometry, conductivity) that show relatively small $(1 \%-10 \%)$ changes, the specularity of reflection provides a more sensitive and definitive indication of the solid-liquid phase transition. Data will be presented that support the hypothesis that specularity changes indicate melt in a way that can be measured easily and unambiguously.
\end{abstract}

Keywords: tin, melt, phase change, diagnostic, PDV

PACS: $62.50 .+\mathrm{p}, 47.40 . \mathrm{Nm}, 78.68 .+\mathrm{M}$

\section{INTRODUCTION}

The identification and characterization of phase changes in shock wave experiments are important for equation of state (EOS) determination, but accurate phase change data are difficult to obtain. Attaining high pressures often requires dynamic shock wave experiments, and phase changes are even more difficult to diagnose dynamically than statically. We undertook this work with the hope of developing a simple diagnostic, which would corroborate the occurrence of a dynamic phase change in shock wave experiments in metals. We chose to concentrate on tin because it has both solid-solid and solid-liquid phase transitions that are relatively well known and easy to obtain with common shock wave techniques using guns or explosives. ${ }^{1,2}$ Tin exists in its tetragonal $\beta$ state at standard temperature and pressure, transforms to a body-centered tetragonal (bct) $\gamma$ state at $9.4 \mathrm{GPa}$, and melts at $49 \mathrm{GPa}$. Melt can also be achieved by shocking tin to above roughly $22 \mathrm{GPa}$ and allowing the shock to release into air or vacuum.

Several properties intrinsic to metals - crystal structure, density, conductivity, sound speed, reflectivity, yield strength, and others - depend on material phase. Phase changes often result in a density change as the atoms transform from one crystal lattice type to another with a different packing efficiency. Such a change in density and lattice structure may be observed either through radiographic density measurements or by using $\mathrm{x}$-ray crystallography. ${ }^{3}$ However, in dynamic experiments, x-ray techniques can be difficult to apply to metals because of their high mass-attenuation coefficients and random crystal grain orientations.

\footnotetext{
${ }^{1}$ Electronic mail: stevengd@nv.doe.gov
} 
Another way to determine whether melting has occurred is by measuring the sound speed. As a metal melts it loses its ability to support shear, and consequently the sound speed of the liquid drops. McQueen et al. ${ }^{4}$ developed a technique to measure the melt curve using this phenomenon, but their technique requires several experiments at impact velocities above and below melt. Each experiment uses samples of several step thicknesses, with optical analyzers required for each step in each experiment.

The reorganization of atoms within a crystal lattice can also change a material's conductivity and optical properties. Most metals remain conductive throughout their various phases. Generally only small changes in conductivity are observed, and the direct measurement of such changes in shock experiments can be difficult. A change in the conductivity of a metal can cause accompanying changes in its optical properties, such as the reflectivity. For example, at standard temperature and pressure, tin is in the metallic $\beta$, or "white" phase, and reflects light well. Below $13^{\circ} \mathrm{C}$ tin exists in the $\alpha$, or "grey" phase, and is a semiconductor. In this "grey" phase tin is less reflective. At pressures above $9 \mathrm{GPa}$, tin enters the bct $\gamma$ phase, which has a higher density than the $\beta$ phase. Partial melt can be reached when a shock above approximately 22 GPa releases into vacuum or air. At higher pressures, the melt fraction increases with shock pressure. Complete melting is reached at $49 \mathrm{GPa}$. Elias et al. ${ }^{5}$ studied reflectivity changes of tin to determine the melt-on-shock-release threshold using a Fabry-Perot interferometer. They observed a large drop in the returned Fabry-Perot light levels upon melt. A group at Lawrence Livermore National Laboratory ${ }^{6}$ is developing a technique to determine phase changes using ellipsometry. Both of these methods presently require large, complex diagnostics.

Velocity profiles from optical velocimetry techniques sometimes suggest melt. Measurements of the free surface velocity of tin as a function of time (wave profile measurements) are known to become difficult above melt. Holtkamp et al. have published VISAR wave profiles for tin shocked from below ${ }^{7,8}$ up to slightly above the melt threshold. ${ }^{8}$ For laser-shocked tin, de Resseguier et al. ${ }^{9}$ have published VISAR data showing very similar features. In these experiments, as well as in many others, the VISAR signals disappear at melt or soon after. Although the signal drops, there is often still adequate light intensity for a VISAR signal. It is usually assumed that these problems are an indication of multiple Doppler frequencies (corresponding to multiple material velocities) as most VISAR systems require a very narrow optical wavelength range to function. Our own VISAR observations for melt-on-release show that the signal typically vanishes within a nanosecond of shock breakout (SBO). Although a Fabry-Perot interferometer is capable of producing wave profile data in the presence of multiple surface velocities, velocity data are not given in Ref. 5 .

Recent advances in high-bandwidth recording and high-speed optical communications technologies have allowed researchers to avoid some of the problems with VISAR and Fabry-Perot velocimetry by using a homodyne interferometer. This device mixes reflected laser light that has been Doppler shifted by the sample's surface motion with unshifted light from the same laser. The instrument, a Photon Doppler Velocimeter (PDV), ${ }^{10}$ uses a detector and digital recorder fast enough to respond to the optical 
difference frequency (or beat frequency) between the two signals. Sliding short-time Fourier transforms can be used to identify the Doppler-shifted velocities. The laser wavelength in such measurements is typically $1.5 \mu \mathrm{m}$. A change in displacement of half this amount $(750 \mathrm{~nm})$ results in a change of one cycle in the interferometer signal. A typical PDV is shown schematically in Ref. 10.

A PDV is much better than VISAR at determining velocities from signals containing multiple Doppler frequencies, and it has provided good wave profiles and stress determinations above as well as below melt. For rough surfaces, PDV often indicates the presence of multiple velocities, some of them likely from ejecta ${ }^{11,12}$ produced at SBO. Spectral analysis of PDV signals extends the dynamic range of the measurements well beyond the dynamic range of a transient digitizer, allowing measurements of these very weak ejecta or backscatter signals from roughened surfaces. For highly polished or diamond-turned surfaces shocked beneath melt, only one velocity-that of the free surface-is observed by PDV.

For tin and other metals the yield strength drops sharply at melt, providing yet another diagnostic opportunity. Examination of recovered shocked samples can sometimes indicate spall damage and/or full or partial melt. In Refs. 8 and 9 it is evident that the spall strength above melt is smaller than that below. Using PDV we have seen that some, but not all, experiments just above the melt threshold show similarly low strength, typically less than $0.3 \mathrm{GPa}$, while the remainder exhibit negligible strength. As the shock stress and melt fraction increase, the strength becomes too small for us to measure.

In this paper we report the use of optical backscatter, as well as various radiographic, visible, and infrared (IR) imaging diagnostics, to characterize the melt transition in tin. The results of these studies have enabled us to develop a compact, reliable fiber-optic tool to probe melt on shock release.

\section{EXPLOSIVE PACKAGE}

Shock waves for this work were produced by a high explosive (HE) charge in contact with a tin sample. Typically the HE is a cylinder with a diameter of $12.7 \mathrm{~mm}$ and a thickness of $12.0-12.7 \mathrm{~mm}$. The tin samples ${ }^{13}$ are typically $25 \mathrm{~mm}$ in diameter and $1.5-$ $7.5 \mathrm{~mm}$ thick. The samples and HE are pressed into a Delrin holder. An RP-80 or an RP1 detonator on axis at the back of the HE initiates the detonation, and the resulting byproducts and debris are contained in a steel explosive chamber. A schematic diagram of the explosive and sample appears in the paper by Lutz. ${ }^{14}$ When shocked using $12.7 \mathrm{~mm}$ of PBX-9501 explosive, a 2-mm-thick tin sample will reach a pressure of 28-30 GPa just below the free surface, and it is estimated that the sample will be over $50 \%$ melted when the shock wave releases into air. For a triangular shock wave, which results from an $\mathrm{HE}$ drive, the stress in the sample drops with distance as the wave travels into the sample. By making the tin samples thicker, we obtain lower-peak shock pressures at the free surface, and in this way we can reduce the melt fraction at the surface. Using Detasheet HE, which is less energetic than PBX-9501, the shocked tin remains solid. All the shocks have a small convex curvature at the free surface because of the center detonation of the 
HE, which causes the shock to lead in the middle. Because the samples are larger in diameter than the HE, the shocked region at the center is pushed ahead. Thus there is a region of high strain around $6 \mathrm{~mm}$ from the center, which is at the diameter of the $\mathrm{HE}$ and outside the area of interest for most of our work. There are also rarefaction waves in the sample, originating at the edge of the HE and propagating toward the rear surface of the sample, as well as toward its center, at roughly equal speeds. Consequently, the size of the area that receives a single un-attenuated shock decreases with increasing sample thickness, and we must confine most of our investigations to the center part of the sample.

\section{X-RAY EXPERIMENTS}

Two 20-ns-duration flash x-ray (350-keV endpoint) radiographs taken about $12 \mu$ s after SBO are shown in Fig. 1. The images illustrate the late-time appearance of samples shocked to below and above melt. The slight curvature of the leading edges at the tops of the images shows the non-planarity typical of our experiments. To minimize ejecta, the samples had highly polished surfaces, with an rms deviation from the average plane of the surface of 30-35 nm or less. Ejecta, which would be ahead of the free surface, are not apparent for these two shots. For the experiment below melt [Fig. 1(a)] the shocked and expanded sample is too dense to show significant density variations in the radiograph. Above melt [Fig. 1(b)], there is a conical-shaped, low-density cloud of fine particles in the center. The leading edge of this cloud is the free surface of the sample. The distance the leading edge has traveled is consistent with the free-surface velocity measured with PDV.
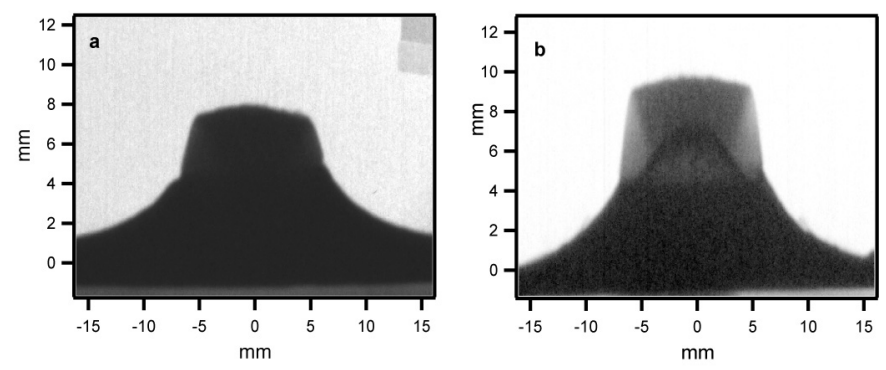

Figure 1. Pulsed (20-ns) x-ray radiographs of two tin samples shocked by 12.7-mm-diameter HE cylinders to pressures below (a) and above (b) melt on release. The $x$-rays illuminated the sample from the side at 11 and $12 \mu \mathrm{s}$, respectively, after shock breakout. The HE was below the samples, out of the images, so the tin is moving upward. Roughly the center third of each image shows tin that was initially directed above the HE, and the leading edge has about the same diameter as the HE. The expanding volume of the melted sample has lower density and travels faster than the solid sample.

At pressures above the phase change, we believe that as the rarefaction from the shock release proceeds backward into the sample (downward in the figure), it melts the tin, beginning at the free surface. ${ }^{8}$ The liquid tin is propelled upward, away from the sample remnant, at about twice the instantaneous particle velocity beneath the surface when the pressure releases. Thus the cloud is being produced with time as the rarefaction front travels into the sample. In contrast, ejecta are assumed to be produced only at SBO time, and their velocity can exceed that of the free surface. ${ }^{11}$ The conical shape of the cloud 
arises as the edge release at the outer diameter of the shock moves radially toward the center of the sample, reducing the diameter of the main shock and the cloud that the shock's reflection produces. Lower-density material appears to the sides of the center cone. This material apparently originates from the edge-release shock, which is lower in pressure than the main shock. As the edge release moves radially, both inward and outward from the original shock diameter, it produces a cloud of lower density than the center cone. The sample remnant appears as a curve near the axis at the bottom of the cloud. At this late time, the sample remnant is considerably more bowed than the leading surface, and it is thin enough that only the limbs of the three-dimensional remnant appear dark in the radiograph. Finally, there is dense material at the lower, outer part of the image. The sample was larger than typical (40 $\mathrm{mm}$ in diameter), but the HE was the standard $12.7 \mathrm{~mm}$ size. The part of the sample that was not directly above the HE has been pushed upward, but it remains solid and thick enough to be opaque in the radiograph.

\section{OPTICAL IMAGING EXPERIMENTS}

To investigate changes in surface characteristics upon melt, we performed two types of optical imaging experiments, first using a high-speed framing camera ${ }^{15}$ and subsequently a higher resolution, single-frame camera. ${ }^{16}$ These experiments recorded images of shocked-tin samples and indicated that the surface changes character greatly upon melt. Below melt, 100-ns-duration framing-camera images of a flash lamp, reflected from the polished surface, remained nearly undistorted for many microseconds. Above melt, the flash lamp images disappeared almost immediately upon SBO.

High-resolution, single-frame images of samples taken below and above the melt on release threshold are shown in Fig. 2(a) and Fig. 2(b), respectively. The samples are sideilluminated, and in both cases the spatial resolution is approximately $40 \mu \mathrm{m}$. The image in Fig. 2(a) was taken 900-910 ns after SBO and shows very little change in the surface, with most of the surface remaining specular (dark in the picture). In contrast, the above melt on release image taken 550-570 ns after SBO [Fig. 2(b)] shows that the surface quickly became roughened, with few specular (dark) areas remaining.
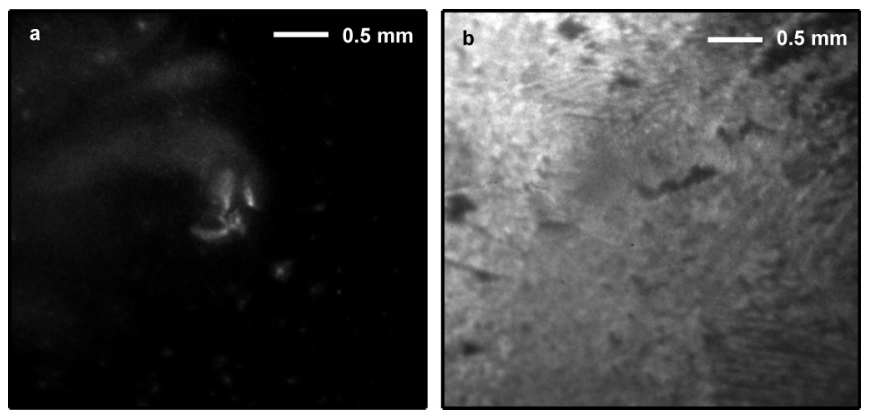

Figure 2. High-resolution images of shocked tin taken below (a) and above (b) the threshold for melt on release of the shock wave. A xenon flashlamp illuminated the samples from the left. Bright regions in both images correspond to diffusely scattered light reaching the camera; specular regions remain dark. The center region of image (a) is flash lamp light reflecting from the convex surface at the center of the shocked sample. Both images were taken shortly after shock breakout. The spatial 
resolution of both images is approximately $20 \mu \mathrm{m}$. The brightness of image (a) was doubled in order to make the image features visible. The signal level in image (a) is nearly zero in most regions, consistent with a smooth surface finish.

In a sense, the images in Figs. 1 and 2 are of the same phenomena, but they view the sample from different angles and at different times. Figure 1 is taken from the side, and Fig. 2 is taken along the axis. The density of the cloud above melt is high enough that the optical path, even at the leading edge, is at most a few tens of microns; therefore the flash lamp light does not penetrate very far. Consequently, Fig. 2 shows the leading edges of the shocked samples as early-time surfaces.

We also took 500-ns-duration IR images of the shocked surface to determine the uniformity of the IR spectral radiance. ${ }^{17}$ The technique has been described previously by Lutz et al. ${ }^{14}$ These IR images also showed marked differences depending on the melt state. Below melt, the spectral radiance varied gradually from center to edge of the shocked region. For shock pressures of 24-25 GPa, which are just above melt, there appeared a few high-radiance spots, usually near the edge of the shocked region, where the deformation of the sample was likely greatest. For pressures around $30 \mathrm{GPa}$, the shocked region appeared as a mixture of many small regions of high and low radiance, comparable in physical size to those recorded in the visible image in Fig. 2(b).

It is instructive to compare our high-resolution visible images with proton radiographs (pRad images) such as those in Ref. 8. The pRad images of shocked, free-surface tin were taken for a pair of explosive-driven shocks, one below and one just above free surface melt on release (see Figs. 8 and 10 of Ref. 8.) The material was imaged from the side (normal to the shock axis) at $2.1-\mu$ s intervals using up to 20 pulses of $800-\mathrm{MeV}$ protons synchronized to the cameras. Below melt, there appeared to be several individual spallation layers plus a sample remnant in which there was insufficient tension to cause spall. Above melt, the smoothly varying densities are described as resulting from cavitation in the part of the sample where there had been spallation of melted material. The cavitated material formed an expanding particle cloud much like that on the right in Fig. 1. From the sequence of images over a period of $40 \mu \mathrm{s}$ (described, but not shown, in Ref. 8), it was apparent that the decaying nature of the pressure behind the triangular shock wave from the HE results in a distribution of axial velocities in the cloud, fastest at the leading edge and slowest at the sample remnant. The image sequence also illustrates better than the single x-ray radiograph [Fig. 1(b)] that the cloud is not expanding noticeably in the radial direction. Consequently, any radial component of velocity after SBO is small, less than a few meters per second.

\section{OPTICAL SCATTERING EXPERIMENTS}

The results of the x-ray radiography and optical imaging experiments indicate a significant change in surface topography with melting. A set of laser scattering experiments were performed to further characterize this phenomenon. Figure 3 shows the experimental configuration in which the surface of a polished tin sample was positioned at the focal point of an $\mathrm{f} / 1$ Fresnel lens. Since the experiments are destructive, we chose a plastic Fresnel lens to reduce cost and minimize shrapnel damage from the explosion. 
Light from a 532-nm laser was focused through the lens to a spot on the tin, and we looked for any changes in the angular dependence of the reflected laser beam by imaging the lens surface. A framing camera imaged the Fresnel lens surface. For a shot below melt [Fig. 4(a)], the reflected laser spot remained almost unchanged for many microseconds. When the shock wave melted the tin, the reflected intensity in the central spot dropped significantly and the Fresnel lens filled with scattered light, as seen in Fig. 4(b). We concluded that, upon melt, the tin surface suddenly changes from specular to diffusely scattering, and as a result the reflected laser light fills the Fresnel lens.

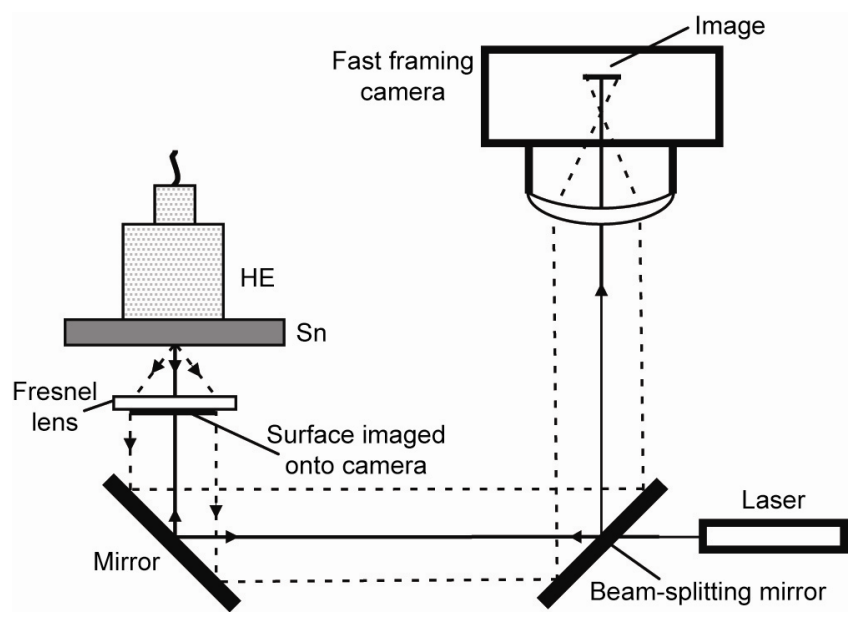

Figure 3. Schematic of Fresnel lens experiment. A tin sample is placed with its surface at the focal point of the lens. Light from a laser (solid line) is reflected (solid line) or scattered (dashed lines) from the tin. Mirrors relay the image of the lens out of the explosive-containment vessel to a high-speed framing camera, which images the lens at nine points-in-time bracketing shock breakout.
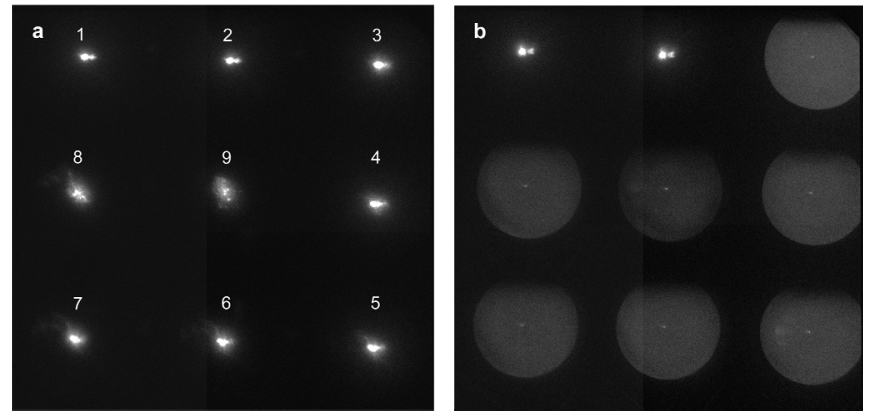

Figure 4. Framing camera images of a Fresnel collection lens indicate specular reflection below melt (a) and diffuse reflection above melt (b). The lens was positioned so that the tin sample is at its focal point, as shown in Figure 3. The smaller, more intense spots in both images are the specular reflection of a 532-nm laser beam focused to a spot on the free surface of a tin sample. The seven large spots in (b) show the lens filled with diffusely scattered light when the tin melts. Frame intervals are $300 \mathrm{~ns}$, proceeding clockwise (labeled 1-9) beginning at upper left and ending in the center. In both experiments, shock breakout occurred between frames 2 and 3.

When we repeated the experiment with polarized laser light and a crossed polarizer covering half of the Fresnel lens, we found that the post-melt scattering effectively depolarized the laser illumination. We took this depolarization as evidence that, after 
melt, most of the reflected light scattered more than once at the surface because depolarization occurs only for deeply rough surfaces or volume scatterers. Finally, we repeated the measurement with a streak camera, and found that the rise-time of the effect was less than the 50-ns resolution of the camera. The streak camera sweep time (and hence the resolution) for this measurement was limited because we had several hundred nanoseconds of timing jitter in the SBO.

\section{FIBER-OPTIC PROBE}

To simplify the diagnostic, we measured the high-angle scattering without imaging. For this purpose, and to improve the time response, we designed a probe that used a $400-\mu \mathrm{m}-$ diameter, step-index optical fiber ${ }^{18}$ to send laser light to and collect light from the sample. The quartz probe fiber was about 4-m long and had a numerical aperture (NA) of 0.48.

Figure 5 shows a schematic.
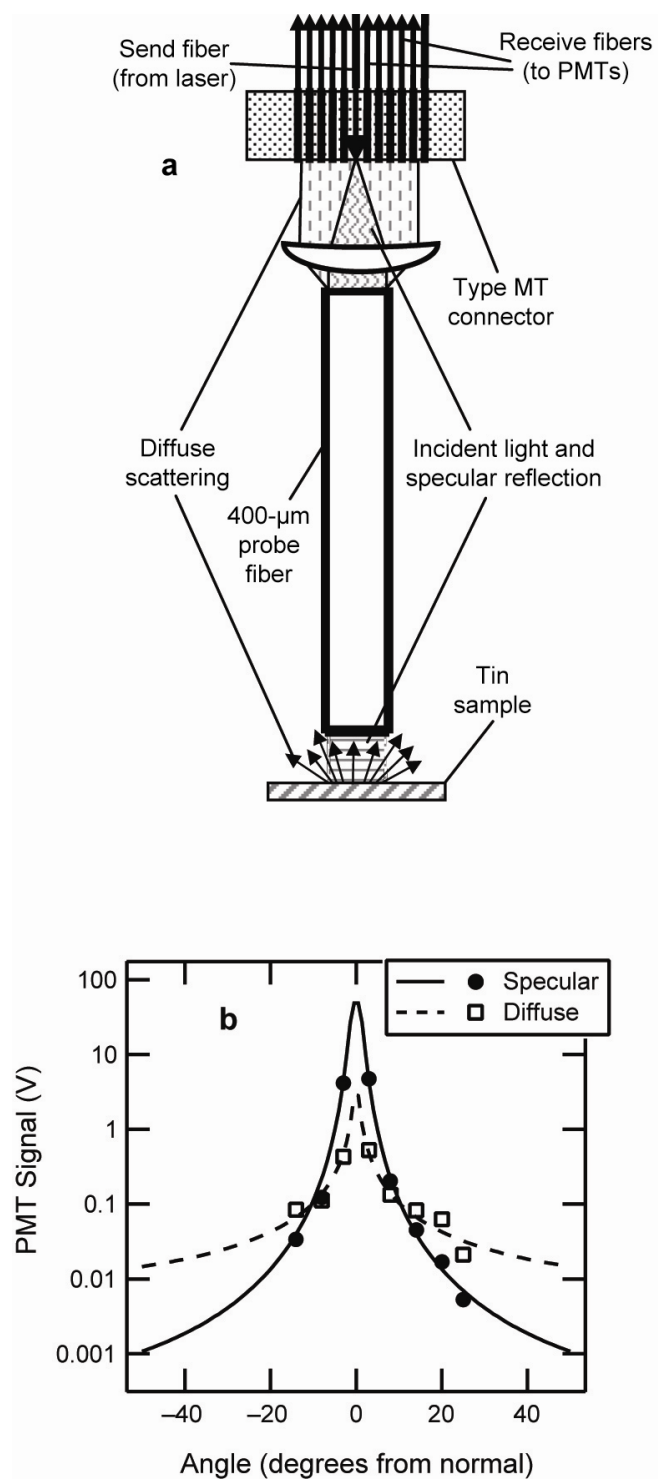
Figure 5. Top: Schematic of fiber-optic probe. Laser light enters from the top on the central fiber, is collimated into the probe fiber, and strikes the sample. Since the input light is collimated, it fills mostly modes for which the light is nearly parallel to the probe axis. Reflected (specular) light returns to the probe fiber nearly collimated and makes a relatively small spot on the fiber-optic connector, lighting up only the receive fibers nearest the send fiber. Scattered (diffuse) light illuminates all the receive fibers. Receive fibers lead to photomultiplier tubes for detection and recording. Bottom: Measured light levels on the receive fibers for a solid shot (dots and solid curve) and a melted shot (open squares and dashed curve). The curves are fits from an arbitrarily chosen Phong scattering distribution.

To test the probe response, we injected laser light at low angles into the center of the fiber core. The other end of the fiber was placed $400 \mu \mathrm{m}$ from a test reflector. By the time the light reached the reflector, it had filled the core, although only the lowest-NA (mostnearly parallel to the axis) modes were filled. Light reflected from a mirror normal to the fiber direction emerged from the injection end nearly collimated. Tilting the mirror produced a light output cone pattern with a half-angle equal to the mirror tilt angle. Reflections from a diffusely scattering surface, such as Spectralon ${ }^{\circledR}$, generated a filled cone of nearly $30^{\circ}$ half angle, which is the NA limit of the probe fiber.

For shocked-tin measurements, we put a lens near the injection end of the fiber to collimate the emerging diffusely scattered light. A linear array of 12 fibers in a type-MT connector was placed across the diameter of the collimated light. One fiber near the center of the array was used to inject laser light into the center of the probe core. Outputs of the four nearest fibers on each side of the injection fiber were monitored with photomultiplier tubes. Specular reflections illuminated the fibers nearest the injection fiber at the center. Large-angle reflections illuminated fibers farther from the center. Diffuse scattering illuminated all the fibers.

Fielding the fiber-optic probe on a pair of shots, one below and one above melt, produced significant differences in the sample specularity (Fig. 5). Upon melt, we found that the two collection fibers nearest the center lost signal, the most distal fibers gained signal, and the collection fibers in the middle had relatively smaller changes. The most definitive results were for the fibers near the center and far from it (i.e., for small and large scattering angles). It was evident that measuring larger angles would be beneficial.

\section{OPTICAL BACKSCATTER DIAGNOSTIC}

To access higher angles, we designed a PDV diagnostic with three fiber-optic probes: one positioned nearly normal to the surface and two at angles of roughly $30^{\circ}$ and $60^{\circ}$ relative to the normal (Fig. 6). Probes consisted of small graded-index collimators placed $8 \mathrm{~mm}$ from the center of the sample surface. Laser light at $1550 \mathrm{~nm}$ was split with fiber-optic couplers and sent into each of the probes on single-mode optical fibers. Scattered light returning to the three probes and fibers was combined in the couplers and sent to the homodyne detector, where it was mixed with unshifted light from the same laser. The three probes illuminated different, slightly offset spots so that they would not interfere with one another. Also, to avoid the large specular signal at $0^{\circ}$, the " 0 " " probe angle was set a bit off normal. Although our sample was polished, the specular return beam was 
broad enough to ensure detection by the $0^{\circ}$ probe without careful alignment. The system required a total of $200 \mathrm{~mW}$ of laser power to obtain adequate return signals both above and below melt. We tested the system before each shot by tapping the sample to be sure that the $0^{\circ}$ probe was sensitive to motion of the reflecting surface. Reflected light from the off-normal probes, which received no backscatter from the specular surface during preshot alignment, was allowed to exit the holder through holes opposite the probes. In the experiment, the observed Doppler shifts for the three angles are proportional to the cosine of the viewing angle times the free surface velocity, as expected. Therefore, in the mixed PDV signal, the light from the three probes can be readily separated because of their distinctly different frequencies. As the surface moves after SBO, the sample spots illuminated by the off-normal probes will move across the surface. This motion provides some information about the uniformity of surface scattering.

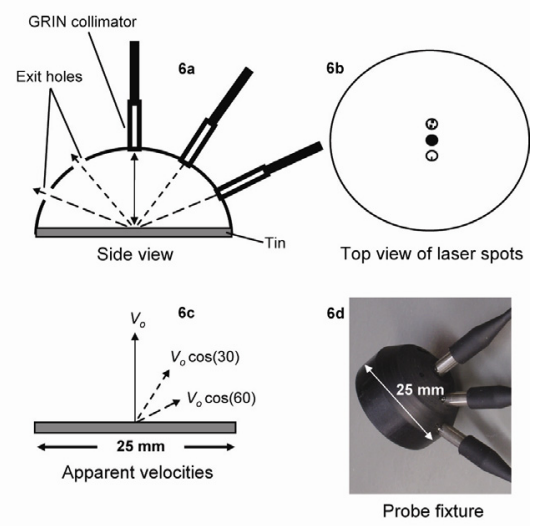

Figure 6. Schematic of backscatter PDV probe. $1.5 \mu \mathrm{m}$ laser light enters the three graded-index-lens collimators on single-mode optical fibers, and the collimators illuminate separate spots on the tin sample. Scattered light is collected in the lenses and returns to a PDV homodyne detector, where it interferes with light from the same laser that is not Doppler shifted. Before melt, little light returns to the off-normal probes, instead exiting through holes in the fixture.

Fourier analysis of three shots produced the spectrograms shown in Fig. 7. The observed optical frequencies are shown as darkened regions that denote velocity vs. time. More intense Doppler frequencies appear darker. Different probes appear at different apparent velocities, because we have not corrected for the detection angle. 

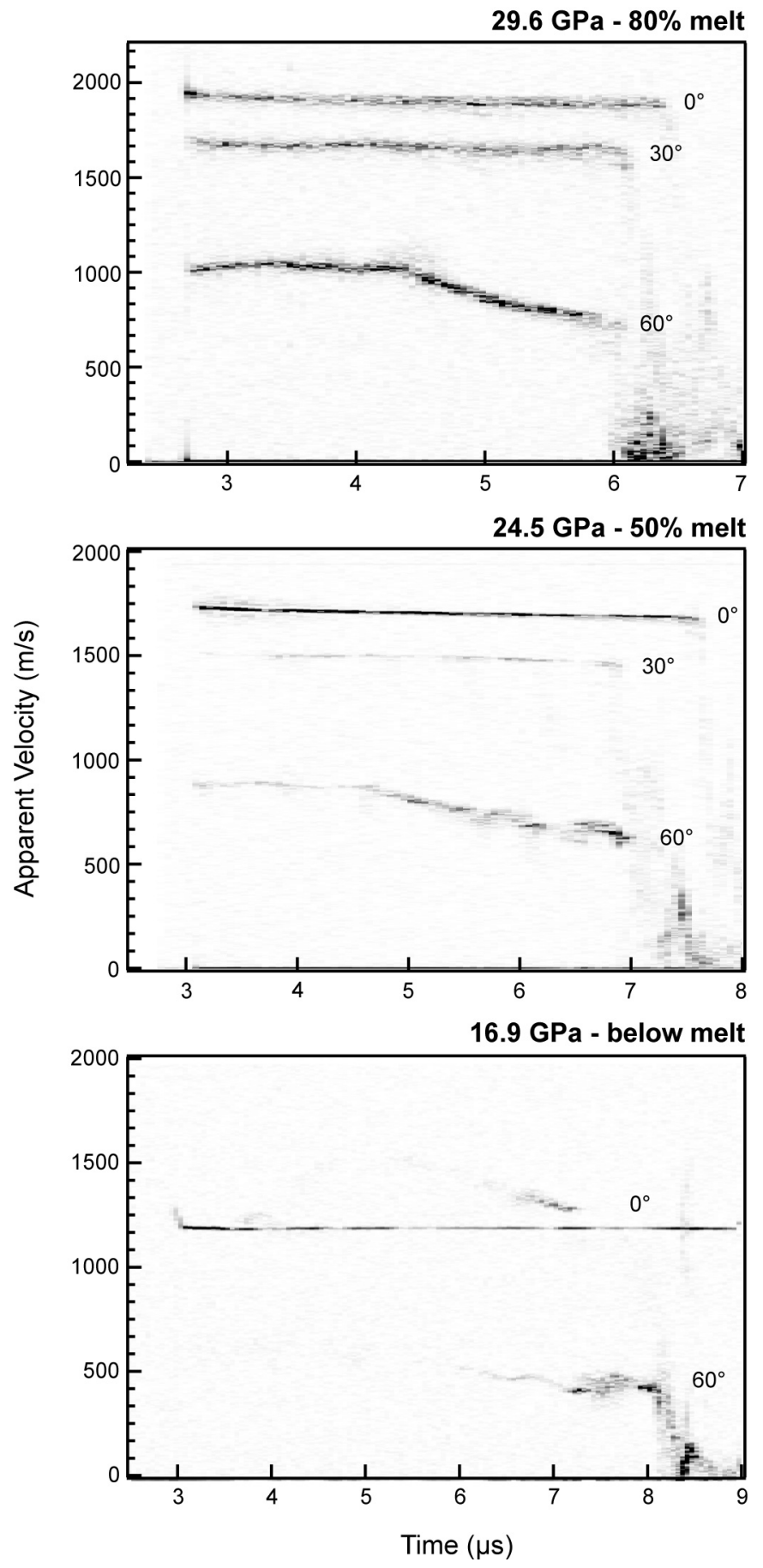

Figure 7. Spectrograms for shocked tin above (upper), slightly above (center), and below (lower) melt on release. The vertical axis is the apparent velocity in meters per second. This quantity is calculated by multiplying the detected Doppler frequencies by $750 \mathrm{~nm}$, half the wavelength of the laser light.

The horizontal axis is time in microseconds. Three light collection angles are present in each case: $0^{\circ}$, $30^{\circ}$, and $60^{\circ}$. The last two are not visible below melt until the sample has moved far enough for the probes to view its edges. Shock breakouts are at 2.7, 3.1, and $2.9 \mu$ s. Below melt, the lack of signal in the off-normal probes for the first few microseconds indicates a lack of diffuse scattering. 
The upper graph in Fig. 7 shows the results for a 2-mm-thick tin sample shocked using PBX-9501 to melt on release. The three waveforms are identified, from top to bottom, as resulting from backscattering at $0^{\circ}, 30^{\circ}$, and $60^{\circ}$. From the velocity of the top waveform, $1.99 \mathrm{~km} / \mathrm{s}$, we know that the peak sample stress just below the free surface was 29.6 GPa. This signal "turns on" immediately at SBO, when the sample begins to move. The signals from the larger-angle probes, which require the sample to roughen to produce large-angle scattering, often appear to turn on within a few nanoseconds of SBO. The sample strikes the lowest $\left(60^{\circ}\right)$ probe about $1.5 \mu$ s after SBO.

To understand the melt conditions, we ran numerical simulations using the code WONDY $^{19}$, with a three-phase EOS subroutine written by Hayes ${ }^{20}$, and the EOS parameters of Ref. 1 . To partially compensate for the two-dimensionality of our problem while keeping the geometry unchanged, we reduced the energy of the HE drive until we matched the observed free-surface velocity. These calculations predict that for these conditions the tin was about $80 \%$ melted near the surface.

The center graph of Fig. 7 is for a 3-mm-thick tin sample. Here, the free surface velocity is $1.73 \mathrm{~km} / \mathrm{s}$ and the shock stress is $24.5 \mathrm{GPa}$, slightly above the predicted melt threshold. The numerical simulations predict a melt fraction of about $50 \%$ near the surface. At early times, the off-normal probes returned relatively weaker signals than at higher stresses, but they are still unambiguously present.

The bottom graph is for a 2-mm-thick tin sample shocked with Detasheet to a stress of 16.9 $\mathrm{GPa}$, somewhat below melt. The $0^{\circ}$ signal shows a velocity drop from 1.30 to $1.21 \mathrm{~m} / \mathrm{s}$ immediately after SBO, corresponding to a change in stress from 16.9 to 15.5 GPa. This change signifies that the material had some strength, of order $0.7 \mathrm{GPa}$ (half the difference between the peak and minimum stresses), and the sample was not melted. The $30^{\circ}$ probe signal is not visible, and the $60^{\circ}$ signal only becomes visible about $3 \mu \mathrm{s}$ after SBO when it is impacted by the moving surface. We interpret these signals as evidence that the center of the surface did not become diffusely reflecting at SBO, as was the case above melt.

\section{DISCUSSION}

For tin shocked above about $22 \mathrm{GPa}$ but below $49 \mathrm{GPa}$, the isentrope for release from the shocked state into air or vacuum crosses the bct-liquid boundary. ${ }^{1}$ The rarefaction from the shock reflection releases the stress in the sample to atmospheric pressure, melting the tin at that point and setting the liquid in motion at about twice the particle velocity that existed in the sample at the melt location. This melt begins at the free surface and proceeds backward into the sample as the rarefaction moves into the sample. Since it lacks strength, the tin forms a cavitated cloud, which produces a free surface that is much less smooth than the unshocked sample or a sample that remains solid after shock release. It should be emphasized that the features reported as "cavitated" material are different from ejecta, which are usually thought of as small particles or jets originating from defects in or near the surface of the experimental sample at SBO and traveling faster than the free surface. Instead, the leading edge of the cavitated region is what is being 
observed by PDV and the visible imaging as the free surface of the sample after shock release.

Not apparent in Fig. 7 is the observation that the spread in the measured Doppler velocities is much greater above melt than below. By repeating the Fourier analysis with larger time bins, we can estimate the inherent frequency widths of the PDV waveforms and therefore also the reflecting-surface velocity spreads. Figure 8 shows the results for several experiments, ranging from 17 to $32 \mathrm{GPa}$. At 29-32 GPa, the velocities in a given PDV waveform have a range of $\sim 20 \mathrm{~m} / \mathrm{s}, 1 \%$ of the free surface velocity. This is easily capable of destroying the phase coherence of the reflected beam enough to disrupt a VISAR signal. Loss of coherence is also consistent with the observed loss of polarization. However, the velocity spread is not enough to be observable in Fig. 1 with our x-ray radiograph resolution, which is 2 line pairs $/ \mathrm{mm}$. For shots below $23 \mathrm{GPa}$, the tin retains its strength until it spalls, and the free surface expands as a unit. PDV cannot penetrate beyond the first spall layer, and the velocity spread is small.

Two observations are relevant. First, the spectrogram line widths fluctuate in time. The error bars in Fig. 8 represent the standard deviation of the fluctuations in the FWHM over the first recorded microsecond. Second, for high-pressure shots (above $26 \mathrm{GPa}$ ), the $30^{\circ}$ and $60^{\circ}$ waveforms have approximately the same width as the $0^{\circ}$ waveforms. A possible interpretation is that the reflecting material at the free surface has about as much variation in transverse velocity as in the forward direction. The melt shot of Fig. 10 of Ref. 8 was at a lower stress. For it, the cavitated cloud at $40 \mu \mathrm{s}$ after SBO would have expanded in the transverse direction by about $0.2 \mathrm{~mm}(0.4 \%$ of its initial diameter $)$ or less. Such a small expansion might not be readily apparent in that experiment.

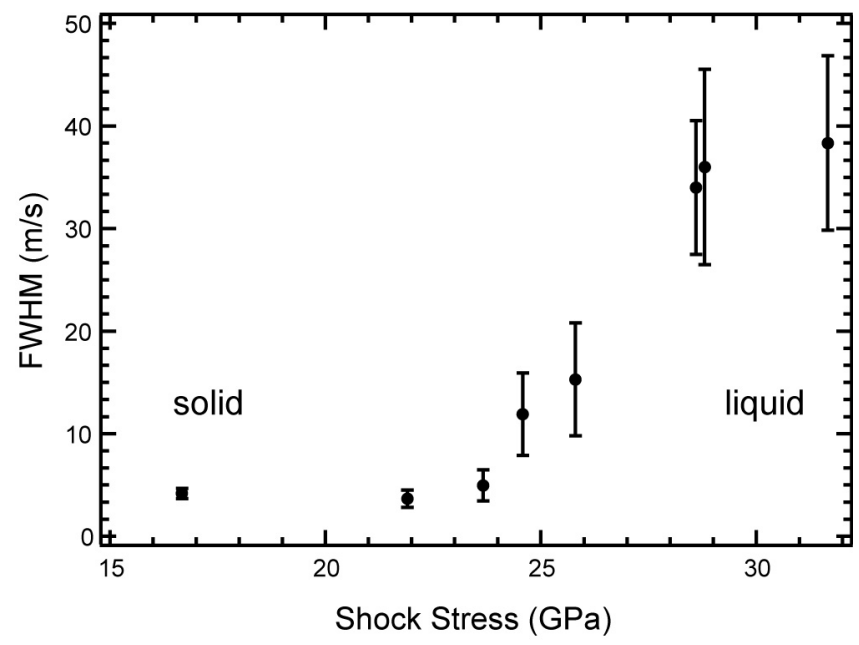

Figure 8. Spread in the measured surface velocities as a function of peak shot pressure below the free surface. Velocity spreads were obtained from the widths of the Fourier transform lines in the PDV experiments. The melt-on-release threshold is at approximately $23 \mathrm{GPa}$.

Factors other than phase-change-induced expansion influence the sample behavior at SBO. (1) The triangular wave for our HE-driven experiments produces tension in the sample upon reflection from the free surface. Below melt, the tension causes spall, and 
the evidence typically appears as a free-surface velocity decrease, or pullback, for 3050 ns after SBO. (2) Ejecta from surface defects are commonly seen for machined samples with rough surfaces. ${ }^{11,12,21}$ Although we used polished samples, which are known to produce few ejecta particles even above melt, we cannot rule out the existence of ejecta traveling less than a few percent faster than the free surface speed. (3) The shock wave for our center-detonated HE has curvature with a radius of 100-200 $\mathrm{mm}$. The transverse tension caused by increasing the length of the surface will pull the surface apart eventually. Images of this process taken with a high-speed framing camera, but not included in this paper, show a few large cracks for a solid sample, and they appear on a relatively longer time scale of many microseconds. We were unable to record similar images above melt because of the changes in surface specularity.

\section{SUMMARY}

We have noted several observations concerning surface changes of tin. These changes occur at pressures above melt on shock release but not for the unmelted case. Most of these changes can be explained by assuming that upon release to atmospheric pressure or vacuum, the tin melts and increases in velocity, forming a cloud with a nearly continuous velocity distribution and a rough surface. Below melt, the expanding tin sample forms spall layers or remains a single piece, depending on pressure, and the surface remains more smooth.

There are several key observations above melt. (1) VISAR signals disappear, although there often remains sufficient light for a VISAR to operate, presumably because of an increase in the range of velocities of the scattering non-uniformities or particles at the surface. (2) PDV velocities spread for a similar reason. The distribution of off-normal PDV frequencies appears to indicate longitudinal and perhaps also transverse velocity distributions at the surface. (3) Ejecta (particles originating at SBO from defects in the surface smoothness and typically having longitudinal velocities between 1 and 2 times that of the free surface) are nearly negligible for highly polished or diamond-turned samples. Ejecta velocity distributions observed with piezoelectric pins and x-rays are consistent with PDV measurements for both polished and rough samples. However, neither pins nor X-radiography can reliably measure ejecta with velocities less than a few percent faster than that of the free surface. (4) High-magnification, high-speed images show many small $(<50 \mu \mathrm{m})$ features and a rough surface above the melt-on-release threshold. IR images show similarly sized features. (5) Reflected laser light scatters from a diffuse surface, and the light is depolarized. Depolarization requires either a volume scatterer, such as a particle cloud, or a rough surface with features deep enough to cause multiple scattering of most of the photons. (6) Late-time x-ray and proton radiography experiments show distributions with features that would likely evolve from what we describe.

We have built a dynamic melt diagnostic based on surface reflectivity changes from specular to diffuse. It is relatively inexpensive and easy to field. When compared to a diagnostic that relies entirely on the disappearance of reflected light, it is unambiguous and definitive. An advantage is that PDV backscatter observes a phenomenon turning on, 
not something disappearing. The diagnostic uses three fiber-optic channels with inexpensive lenses and a holder for them, along with a single PDV detector and recording channel to measure the surface velocities. PDV is rapidly becoming a standard shock diagnostic, and many laboratories have already invested in the hardware (laser and fast transient digitizer) required to field it. The backscattering diagnostic requires additional testing to see how well it will function for planar shocks and for unpolished samples. We have a tentative understanding of its mode of operation derived from these data and believe that the diagnostic may be able to detect melt on release in materials other than tin.

It is evident that the major cause of the reflectivity changes for a free surface at melt, as seen by some past workers, ${ }^{5,22}$ is an increase in large-angle scattering caused by roughening of the surface, not necessarily a large change in the reflectivity of tin as it melts. Recent dynamic reflectivity measurements have been performed using integrating spheres to look for small changes in reflectivity. ${ }^{23}$ However, if the goal is simply to identify melt on release, then the diagnostic described here should accomplish that goal in a far simpler manner.

\section{ACKNOWLEDGMENTS}

We are indebted to Michael Grover for preparing the samples and assembling and fielding nearly all of experiments at the Santa Barbara explosive-containment Boombox and for his innumerable suggestions and improvements to the system. We thank Dennis Hayes, who gave us his three-phase WONDY EOS subroutine for tin and patiently answered our many questions on how to use and interpret it. We also thank the many people who helped with other phases of the experiment, including especially Guy Leach, Greg Macrum, Andrew Montoya, Adam Iverson, William Buttler, Michael Zellner, and Achim Seifter. This work was performed under the auspices of the U.S. National Nuclear Security Agency.

This manuscript has been authored by National Security Technologies, LLC, under Contract No. DE-AC52-06NA25946 with the U.S. Department of Energy. The United States Government retains and the publisher, by accepting the article for publication, acknowledges that the United States Government retains a non-exclusive, paid-up, irrevocable, world-wide license to publish or reproduce the published form of this manuscript, or allow others to do so, for United States Government purposes.

${ }^{1}$ C. Mabire and P. L. Hereil, Shock Compression of Condensed Matter - 1999, Ed. by M. D. Furnish, L. C. Chhabildas, and R. S. Hixson (American Institute of Physics, 2000) pp. 93-96.

${ }^{2}$ W. W. Anderson, F. Cverna, R. S. Hixson, J. Vorthman, M. D. Wilke, G. T. Gray, and K. L. Brown, Shock Compression of Condensed Matter - 1999 (American Institute of Physics, 2000) pp. 443-446. 
${ }^{3}$ P. A. Rigg and Y. M. Gupta, Appl. Phys Lett. 78, 1655-1657 (1998).

${ }^{4}$ R. G. McQueen, J. W. Hopson, and J. N. Fritz, Rev. Sci. Instrum. 53, 245 (1982).

${ }^{5}$ P. Elias, P. Chapron, and B. Laurent, Optics Comm. 66, 100 (1983).

${ }^{6}$ R. Patterson, J. Nguyen, and N. Holmes, "Ellipsometry of shocked crystals: birefringence and relaxation of $\mathrm{LiF}, "$ http://meetings.aps.org/link/BAPS.2005.MAR.B11.8 (2005).

${ }^{7}$ D. B. Holtkamp, D. A. Clark, I. A. Garcia, D. L. Hammon, W. F. Hemsing, M. A. Shinas, K. A. Thomas, M. D. Furnish, M. D. Crain, and C. H. Gallegos, CP706, Shock Compression of Condensed Matter - 2003, Ed. by M. D. Furnish, Y. M. Gupta, and J. W. Forbes (American Institute of Physics, 2004) pp. 473-476.

${ }^{8}$ D. B. Holtkamp, D. A. Clark, E. N. Ferm, R. A. Gallegos, D. Hammon, W. F. Hemsing, G. E. Hogan, V. H. Holmes, N. S. P. King, R. Liljestrand, R. P. Lopez, F. E. Merrill, C. L. Morris, K. B. Morley, M. M. Murray, P. D. Pazuchanics, K. P. Prestridge, J. P. Quintana, A. Saunders, T. Schafer, M. A. Shinas, and H. L. Stacy, CP706, Shock Compression of Condensed Matter - 2003, Ed. by M. D. Furnish, Y. M. Gupta, and J. W. Forbes (American Institute of Physics, 2004) pp. 477-482.

${ }^{9}$ T. de Resseguier, L. Signor, and A. Dragon, J. Appl. Phys. 101, 013506 (2007).

${ }^{10}$ O. T. Strand, D. R. Goosman, C. Martinez, T. L. Whitworth, and W. W. Kuhlow, Rev. Sci. Instrum. 77, 083108 (2006).

${ }^{11}$ W. S. Vogan, W. W. Anderson, and M. Grover, J Appl. Phys. 98, 113508 (2005).

${ }^{12}$ W. T. Buttler, M. B. Zellner, R. T. Olson, P. A. Rigg, R. S. Hixson, J. E. Hammerberg, A. W. Obst, J. R. Payton, A. Iverson, and J. Young, J. Appl. Phys. 101, 063547 (2007).

${ }^{13}$ ESPI Metals, Ashland, OR, 4N tin rod material (99.99\%-purity tin).

${ }^{14}$ S. S. Lutz, W. D. Turley, P. M. Rightley, and L. E. Primas, Shock Compression of Condensed Matter - 2001, Ed. by M. D. Furnish, N. N. Thadhani, and Y. Horie (American Institute of Physics, 2002) pp. 1239-1242.

${ }^{15}$ Bechtel Nevada, Los Alamos, NM, camera model H-FC-08 Nine Frame Camera.

${ }^{16}$ Questar QM-1 (New Hope, PA) telescope and a Barlow 2x lens mated to a Proxitronic (Bensheim, Germany) model MCPII multichannel plate mounted to a CCD camera (PixelVision, Tigard, OR). 
${ }^{17}$ A Seifter, A. Obst, and D. Holtkamp, $15^{\text {th }}$ American Physical Society Topical Conference on Shock Compression of Condensed Matter - 2007, Hawai'i, June 24-29, 2007.

${ }^{18}$ Thorlabs, Newton, NJ, model BFL48-400.

${ }^{19}$ M. E. Kipp and R. J. Lawrence, "WONDY V - A one-dimensional finite-difference wave propagation code," Sandia National Laboratories Report SAND81-0930 (1982).

${ }^{20}$ D. B. Hayes, J Appl. Phys. 46, 3438 (1975).

${ }^{21}$ D. S. Sorenson, R. W. Minich, J. L. Romero, T. W. Tunnell, J Appl. Phys. 92, 5830 (2002).

${ }^{22}$ M. Werdiger, S. Eliezer, S. Maman, Y. Horovitz, E. Moshe, A. Ludmirsky, B. Arad, Z. Henis, and I. B. Goldberg, Laser and Particle Beams 17, 547 (1999).

${ }^{23}$ D. Partouche-Sebban and J. L. Pelissier, Shock Waves 13, 69 (2003). 